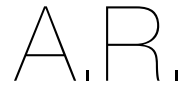

ARTIGO DE REVSÃO

${ }^{1}$ Escola Superior de Tecnologia da Saúde de Coimbra do Instituto Politécnico de Coimbra

Rua 5 de Outubro,

3046-854 Coimbra,

Portugal

*Endereço para correspondência:

Rafaela Lapo

Escola Superior de Tecnologia da Saúde de Coimbra do

Instituto Politécnico de Coimbra,

Rua 5 de Outubro,

3046-854 Coimbra, Portuga

rafaelalapo14@gmail.com

Histórico do artigo

Recebido a 1 de julho de 2021 Aceite a 17 de outubro de 2021

\title{
A INFLUÊNCIA DO GLUCOMANANO NO CONTROLO DE PESO
}

\author{
THE INFLUENCE OF GLUCOMANNAN IN WEIGHT CONTROL
}

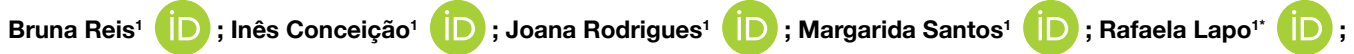

Ana Baltazar ${ }^{1}$ iD

RESUMO

INTRODUÇÃo: O excesso de peso e a obesidade são considerados uma problemática de saúde, podendo ter repercussões na mortalidade e qualidade de vida dos indivíduos. O glucomanano é um componente significante do cormo de konjac, proveniente da planta designada por Amorphophallus konjac. É considerado uma fibra dietética fermentável solúvel, que forma géis, e o seu papel como suplemento alimentar tem sido estudado nos últimos anos de modo a avaliar potenciais benefícios, nomeadamente no controlo ponderal.

OBJETIVOS: Analisar a evidência científica existente sobre a suplementação de glucomanano no controlo de peso, bem como entender os riscos para a saúde inerentes ao seu consumo.

METODOLOGIA: A pesquisa bibliográfica foi realizada nas bases de dados PubMed e ScienceDirect, recorrendo-se às palavras chave "glucomannan" e "weight". Os resultados obtidos incluíram artigos, preferencialmente, dos últimos 10 anos, sendo que inicialmente foram selecionados pelo título, em seguida pelo resumo e por fim pela leitura completa dos mesmos.

RESULTADOS: O glucomanano promove sensação de saciedade devido à sua capacidade de absorção de água, potenciando a motilidade intestinal. A maioria das revisões analisadas verificou uma perda de peso significativa a curto prazo, em adultos com excesso de peso ou obesidade, no entanto um outro estudo com maior número de participantes, não verificou resultados positivos neste aspeto. Em crianças e adolescentes, os estudos são escassos não mostrando efeitos significativos na redução de peso. A dose recomendada com potenciais efeitos benéficos em adultos é de $3 \mathrm{~g} / \mathrm{dia}$.

CONCLUSÕES: Os ensaios futuros devem ter melhor qualidade metodológica. Assim sendo, apesar da recomendação de glucomanano para perda de peso parecer segura, esta não deve ser a primeira abordagem nem uma prioridade.

\section{PALAVRAS-CHAVE}

Glucomanano, Peso

\section{ABSTRACT}

INTRODUCTION: Overweight and obesity are considered major health problems that increase mortality and quality of life. Glucomannan is the significant component of konjac corm, which comes from the plant Amorphophallus konjac. Glucomannan is a soluble, fermentable dietary and natural gel-forming fiber and in the last few years, its role as a dietary supplement has been evaluated to access its potential health benefits, namely weight control.

OBJECTIVES: Evaluate the evidence for or against the efficacy of glucomannan supplement in body weight control and if there's any health risk related to its intake.

METHODOLOGY: Data collection was carried out through scientific databases Pubmed and ScienceDirect, using as research expressions: "glucomannan" AND "weight". The results, preferably from the last ten years, were first analysed by title, then abstract and finally full-text reading.

RESULTS: Glucomannan promotes a "feeling full" effect due to the water-absorbing capacity of the fiber. It also helps intestinal motility. In the short term, most of the reviews found a significant weight loss in overweight and obese adults, although another study with a greater number of participants found no significant weight loss. In children and adolescents, the studies are scarce. However, they show no effect on bodyweight reduction. The recommended dose for potential beneficial effects in adults is $3 \mathrm{~g} / \mathrm{day}$. coNCLUSIONS: Future trials should have better methodological quality. Until then, glucomannan recommendation for weight loss, although it seems safe in most cases, should not be a first approach nor a priority.

KEYWORDS

Glucomannan, Weight 


\section{INTRODUÇÃO}

O crescente interesse na utilização das fibras alimentares para gestão do peso tem gerado, por sua vez, um desenvolvimento de estudos neste âmbito, sendo que as fibras que parecem ser mais promissoras são o glucomanano, quitosana e alguns suplementos que misturam várias fibras (PolyGlycopleX ${ }^{\circledR}$ e Litramine ${ }^{\text {TM}}$ ) $(1,2)$.

O glucomanano é o principal componente do Konjac corm proveniente da planta Amorphophallus konjac, trata-se de uma fibra dietética hidrocoloidal e não iónica, composta por D-manose e D-glucose ligadas por $\beta-1,4$ ligações glicosídicas na razão molar de 1: 1,6-1: 1,4 , dependendo dos genótipos (3). Visto que o glucomanano é uma fibra alimentar solúvel, fermentável e, naturalmente, formadora de gel, este é amplamente utilizado como aditivo alimentar, estando aprovado como tal pela Food and Drug Administration (FDA) (4). Nos últimos anos tem crescido o interesse pela sua utilização como suplemento alimentar para controlo de peso, tendo sido aprovado para este efeito pela European Food Safety Authority (EFSA) em 2010, no contexto de uma dieta hipocalórica para adultos com excesso de peso ou obesidade (5). No Regulamento (UE) N. 432/2012 de 16 de Maio, que estabelece uma lista de alegações de saúde permitidas relativas a alimentos, estão explícitas as condições de utilização, restrição de utilização dos alimentos e/ou declaração/advertência adicional da alegação, sendo que o glucomanano integra esta lista de acordo com o estabelecido pela EFSA no seu documento de opinião científica (6).

$O$ excesso de peso e a obesidade, bem como as suas comorbilidades, atingem milhões de indivíduos em todo mundo, representando proporções epidémicas $(7,8)$. Dados de 2016, reportam que mundialmente existem 1,9 milhões de pessoas com excesso de peso e 650 milhões com obesidade (9). Existem vários tipos de tratamento para a obesidade, sendo o de primeira linha baseado em mudanças de estilo de vida (alimentação e atividade física). No entanto, estas mudanças são, por vezes, difíceis de alcançar e manter, levando à procura de alternativas adjuvantes à perda de peso, como é o caso da suplementação com glucomanano (10). Assim, é cada vez mais utilizada no tratamento para a obesidade e das comorbilidades associadas, como a diabetes mellitus tipo 2, dislipidémias, hipertensão, doenças cardiovasculares e apneia do sono. Por ser uma fibra solúvel, tem um baixo valor calórico e apresenta efeitos benéficos no sistema digestivo, nomeadamente, o aumento da saciedade, o retardamento do esvaziamento gástrico (proporcionado pela capacidade de formar gel) e a diminuição do tempo de trânsito intestinal $(4,7,8,11-13)$.

Normalmente, o glucomanano é comercializado como um suplemento de ingrediente único ou em combinação com outros compostos com características semelhantes associadas ao controlo do peso, como garcinia cambogia, crómio, entre outros $(9,14,15)$.

O objetivo deste trabalho é avaliar a eficácia deste tipo de suplementação no controlo de peso corporal. Adicionalmente, pretende-se perceber se, inerente a este consumo, existem efeitos adversos associados. Para tal, efetuou-se uma pesquisa de artigos científicos nas bases de dados Pubmed e ScienceDirect recorrendo-se às palavras chave "glucomannan AND weight”. Foram obtidos na totalidade 3243 artigos, após ser dada preferência a publicações dos últimos 10 anos, incluindo o ano de 2021 . Apesar deste critério, incluiu-se um artigo de 2010, uma vez que se considerou pertinente para a temática. Após análise do título e resumo e com base em critérios de exclusão, como estudos redigidos num idioma diferente de espanhol, português e inglês e estudos realizados em animais, selecionaram-se 31 artigos. Posteriormente, foi realizada a leitura integral e análise dos artigos selecionados, restringindo a amostra utilizada na elaboração desta revisão a um total de 21 artigos, de acordo com a pertinência para esta temática.

\section{Dosagem}

A dosagem de glucomanano pode variar conforme o objetivo da terapêutica. De acordo com a EFSA, a dose recomendada e aprovada para adultos com excesso de peso ou obesidade é de $3 \mathrm{~g} / \mathrm{dia}$ de glucomanano num contexto de uma dieta hipocalórica que visa a perda de peso corporal, devendo ser consumidas pelo menos 3 doses/ cápsulas, idealmente $1 \mathrm{~g}$ cada, juntamente com 1 a 2 copos de água antes das refeições, para obtenção do efeito reivindicado (5).

\section{Efeito da Suplementação no Controlo de Peso}

A premissa que faz com que o glucomanano seja uma alternativa atraente para promover a perda de peso baseia-se no facto de este ser uma fibra que absorve a água, formando um gel, o que leva ao aumento da sensação de saciedade. Para além disso, o seu peso molecular é elevado, o que permite que seja uma das fibras solúveis mais viscosas, conferindo-Ihe uma vantagem face a outras fibras, já que pode ser consumido em menor quantidade para um mesmo efeito (16).

Uma metanálise recente, de Mohammadpour S et al. (17), reuniu 6 ensaios clínicos randomizados, realizados em adultos (>18 anos) com excesso de peso ou obesidade, que compararam a toma de glucomanano (1,2-3,9g/dia) com um placebo. Excluíram-se estudos que possuíam participantes com doenças crónicas, em que existia suplementação do glucomanano concomitantemente com outros compostos, ou em que a suplementação estava aliada a uma dieta restrita em energia. Obteve-se uma amostra de 255 participantes de ambos os sexos e o tempo de intervenção variou entre 5 e 12 semanas. Os resultados indicaram que existiu uma significativa, mas baixa redução de peso corporal em comparação com o grupo de controlo, sendo que a diferença média do peso entre os grupos foi de 0,96kg. Os melhores resultados foram obtidos em mulheres $(-1,86 \mathrm{~kg})$ e em estudos com duração inferior a 8 semanas (-1,34kg) (17). Um outro estudo de carácter semelhante também procurou avaliar esta relação. Foram incluídos 9 ensaios clínicos randomizados duplo-cegos, sendo que como critério consideraram que o peso e/ou índice de massa corporal (IMC), tinham de estar reportados, e excluíram-se artigos que possuíam suplementação concomitante com outros suplementos (18). Os participantes perfizeram um total de 273 indivíduos de ambos sexos, o tempo de intervenção variou de 3 a 12 semanas e a dose de glucomanano administrada de 1 a 3,9 g/dia, sendo que todos os estudos incluídos apresentaram pelo menos uma forma de controlar os fatores associados ao estilo de vida (18). Dado que um dos artigos incluídos na revisão sistemática não apresentava os dados estatísticos necessários, foi apenas realizada a metanálise com 8 dos artigos incluídos, que indicou que existia uma diferença que não era estatisticamente significativa na perda de peso $(-0,22 \mathrm{~kg})(18)$.

Zalewski et al. (8) avaliaram o efeito do glucomanano em crianças e adultos, excluindo estudos onde os participantes tivessem patologias que exigissem terapia farmacológica. A amostra deste estudo incluiu 60 crianças e 233 adultos de 6 ensaios distintos. A dose de suplemento de glucomanano variou entre 1,24 a 3,99g/dia e o tempo de intervenção de 5 a 12 semanas. A curto prazo a suplementação em adultos pareceu reduzir o peso corporal, mas não o IMC e, nos estudos em que a composição corporal foi considerada, não foram encontradas diferenças significativas entre o grupo de controlo e o grupo onde foi realizada a suplementação. Contudo, em crianças a informação era limitada, pois apenas foi incluído um estudo realizado nesta faixa etária sem resultados significativos, pelo que os autores consideraram a informação insuficiente para gerar conclusões (8). No entanto, anos mais tarde, num outro estudo de Zalewski BM et al., (12) a avaliação dos efeitos do glucomanano em crianças e adolescentes com excesso de peso ou 
Tabela 1

Resumo dos resultados do glucomanano na perda de peso

\begin{tabular}{|c|c|c|c|c|}
\hline AUTORES & AMOSTRA & $\begin{array}{l}\text { DURAÇÃO } \\
\text { (SEMANAS) }\end{array}$ & $\begin{array}{l}\text { DOSE } \\
(\mathrm{G} / \mathrm{DIA})\end{array}$ & $\begin{array}{l}\text { DIFERENÇAS DE PERDA DE PESO ENTRE O GRUPO SUPLEMENTADO } \\
\text { COM GLUCOMANANO COMPARADO AO GRUPO DE CONTROLO }\end{array}$ \\
\hline $\begin{array}{l}\text { Mohammadpour S. } \\
\text { et al (17) }\end{array}$ & 225 adultos & $5-12$ & $1,2-3,9$ & $0.96 \mathrm{~kg} ; 95 \% \mathrm{Cl}:-1.81,-0.11, \mathrm{P}=0.02$ \\
\hline Onakpoya I. et al (18) & 273 adultos & $3-12$ & $1-3,9$ & $\begin{array}{c}\text { Metanálise de } 8 \text { ensaios clínicos revelou uma diferença } \\
\text { estatisticamente não significativa entre os grupos } \\
\qquad-0.22 \mathrm{~kg} ; 95 \% \mathrm{Cl},-0.62,0.19\end{array}$ \\
\hline \multirow{6}{*}{ Zalewskiet B. et al (8) } & 47 adultos & 2 & 3,99 & $-0.21 \mathrm{~kg} ; 95 \% \mathrm{Cl},-0.13,-0.29$ \\
\hline & 20 adultos & 4 & 3 & - 2.04kg; 95\% Cl, $-0.52,-3.56$ \\
\hline & 52 adultos & 5 & 1,24 & $-1.3 \mathrm{~kg} ; 95 \% \mathrm{Cl},-0.89,-1.71$ \\
\hline & 67 adultos & 8 & $3,99-3$ & $-3.17 \mathrm{~kg} ; 95 \% \mathrm{Cl},-1.29,-5.05 / \mathrm{sem}$ diferenças \\
\hline & 29 adultos & 12 & 3 & $+0.1 \mathrm{~kg} ; 95 \% \mathrm{Cl},+1.96,-1.76$ \\
\hline & 60 crianças & 12 & 2 & Sem efeito significativo \\
\hline Zalewski BM et al (12) & $\begin{array}{l}81 \text { crianças e } \\
\text { adolescentes }\end{array}$ & 12 & 3 & $0,0 \mathrm{~kg}$ \\
\hline
\end{tabular}

Números negativos indicam diminuição do peso $(\mathrm{kg})$

obesidade também foi averiguada através da realização de um ensaio clínico randomizado duplo-cego, que incluiu um total de 81 crianças e adolescentes entre os 6 e os 17 anos, sendo que foram excluídos indivíduos com terapia farmacológica para doenças crónicas, histórico de tratamento cirúrgico de obesidade e que participaram em outros estudos de caráter semelhante nos 3 meses anteriores à realização deste. Durante 12 semanas, os participantes receberam glucomanano ou um placebo, ambos em doses de $3 \mathrm{~g} / \mathrm{dia}$, sendo que foram oferecidas consultas com nutricionistas, de modo a atingir uma dieta normocalórica. Para além disso, eram igualmente encorajados a completar 60 minutos de atividade física e um tempo de atividade sedentária inferior a 2h/dia. Neste estudo, não foram encontradas diferenças significativas no IMC para a idade entre a toma de glucomanano e de placebo $(0,0 \mathrm{~kg})$ e a composição corporal também não se alterou nos grupos (12).

Os resultados do glucomanano na perda de peso encontram-se descritos na Tabela 1.

\section{Efeitos Adversos do Consumo/Suplementação}

O glucomanano facilita a formação de géis devido à sua capacidade de absorção de água, pelo que, em raras circunstâncias, esta fibra pode ficar obstruída no trato gastrointestinal, podendo causar engasgamento ou bloqueios potencialmente fatais. Além disto, também pode causar problemas gastrointestinais, como fezes amolecidas, obstipação, flatulência, desconforto e distensão abdominal. No entanto, se se reduzir a quantidade administrada de glucomanano, estes sintomas podem ser atenuados. No caso de o desconforto gastrointestinal persistir, a sua administração deve ser interrompida $(2,3,18,19)$.

\section{ANÁLISE CRÍTICA}

Com base na revisão de literatura efetuada, é possível afirmar que a suplementação de glucomanano nunca deve ser adotada como tratamento de primeira linha, mas sim como uma possível estratégia coadjuvante após terem sido aplicadas mudanças de estilo de vida como primeira abordagem. É ainda necessário ter em consideração que, as perdas de peso estimadas nas revisões que obtiveram resultados positivos $(8,17)$, apesar de significativas, são baixas, o que poderá levantar questões relativamente à sua utilidade clínica.

Relativamente ao uso de suplementação de glucomanano em crianças e adolescentes, os resultados encontrados são escassos e não parecem indicar benefícios $(8,12)$. Desta forma, o seu uso na população pediátrica é desaconselhado e é necessária a realização de mais estudos nesta faixa etária, de modo a compreender a eficácia da sua utilização e os possíveis efeitos adversos.
Os resultados obtidos devem ser interpretados com cautela, dado que os estudos disponíveis atualmente possuem várias limitações. A duração das intervenções é de 3 a 12 semanas, o que é considerado um período curto para testar a eficácia e os efeitos adversos a longo prazo deste suplemento $(8,12,17,18)$. Para além disto, as doses utilizadas nos diferentes estudos $(8,12,17,18)$, analisadas nos resultados, possuem uma variação de 1 a 3,9 g/dia, sendo que a falta de uniformização da dose pode também ser um fator que influencia os resultados obtidos nos ensaios clínicos realizados. Na metanálise de Onakpoya et al. (18), foi observado que não existia uma relação linear entre a perda de peso e a dose de glucomanano fornecida, no entanto a partir dos $3 \mathrm{~g} / \mathrm{dia}$ pareciam ocorrer perdas de peso mais elevadas, o que corrobora a informação disponibilizada pela EFSA $(5,18)$. Nos estudos de revisão avaliados $(8,12,17,18)$, são englobados ensaios clínicos com formas distintas de controlar os fatores associados ao estilo de vida, pelo que seria prudente que os ensaios realizados futuramente procurassem reportar a ingestão energética diária dos participantes e o seu tempo de atividade física diária de modo uniforme (18). Outra das limitações pode ser o facto de os estudos incluídos nas revisões supramencionadas (8, $17,18)$, na sua maioria, não considerarem questões relacionadas com as mudanças da composição corporal, nomeadamente oscilações de massa isenta de gordura e massa gorda, o que pode influenciar os resultados obtidos (20). No que toca ao uso de suplementos, outra das questões que se pode salientar é o facto do financiamento dos ensaios clínicos ser suportado pela indústria. Contudo, Onakpoya l. et al. verificaram que ao incluir apenas estudos financiados pela indústria na sua metanálise, a diferença entre as perdas de peso não foi significativa entre os grupos e o inverso sucedeu ao incluir apenas os estudos que não tinham sido financiados pela mesma (18).

A variabilidade genética também é um fator a ter em conta quando se analisa a perda de peso per si (14). Num estudo de Maia-Landim A. et al. (14), a suplementação de 214 indivíduos, com excesso de peso ou obesidade, com 500mg, 2 vezes ao dia, de Garcinia cambogia e de glucomanano gerou perda de peso e de massa gorda e um aumento da taxa metabólica basal ao final de 6 meses de suplementação. Estes resultados foram independentes da idade, sexo, ou presença de doenças crónicas, no entanto este efeito parece ser atenuado em pessoas com os polimorfismos PLIN4 (Perilipin-4) (11482G>A), ADRB3 (B-adrenergic receptor 3) (Trp64Arg) e FTO (Fat Mass and Obesity associated) (rs9939609 AVT) (14).

Futuramente, é expectável que surjam ensaios clínicos com melhor qualidade metodológica ou novas utilidades para o glucomanano. Numa revisão sistemática de Ejtahed et al. (21) foi estudada a influência 
da manipulação da microbiota intestinal na obesidade, em que a suplementação com glucomanano gerou um aumento de bactérias Bifidobacterium e Lactobacillus e uma diminuição das Clostridium. Esta relação poderá vir a ser explorada, dado que a microbiota intestinal tem sido apontada como um fator presente na etiologia da obesidade e a suplementação com glucomanano pode constituir um fator modificador da mesma (21).

O papel do nutricionista perante a recomendação desta suplementação assenta na priorização dos fatores individuais e socioeconómicos inerentes ao utente, devendo informá-lo acerca da fraca qualidade da evidência atual e que nos estudos que apresentam uma perda de peso significativa, esta é baixa relativamente à perda com a toma de um placebo, podendo aferir pouco significado clínico. Para além disso, o utente também deve ser notificado acerca dos possíveis efeitos adversos associados, ainda sem gravidade relatada, antes de implementar este tipo de suplementação. É ainda necessário ter em consideração que a utilização deste suplemento está contraindicada em indivíduos que possuam algum problema esofágico pré-existente. Nos restantes, a toma deste suplemento deve ser deliberada consoante a existência prévia de problemas gastrointestinais, e perante esta condição deverá ser reduzida a dosagem ou interrompida caso os mesmos surjam após o início da suplementação $(2,3,18,19)$.

Tendo em conta as considerações supramencionadas e a aprovação da EFSA relativamente ao uso do glucomanano, acrescenta-se que seria pertinente rever a literatura científica publicada após 2010, de modo a garantir que os pareceres emitidos pelas entidades competentes estão alinhados com a evidência atual.

\section{CONCLUSÕES}

A suplementação com glucomanano não deve ser adotada como tratamento de primeira linha, apesar de parecer segura, dado que os estudos existentes carecem de qualidade metodológica. No entanto, à luz da evidência científica existente, os resultados demonstram, maioritariamente, perdas de peso estatisticamente significativas em adultos com excesso de peso ou obesidade. É necessário um maior número de estudos de melhor qualidade, de modo que o aconselhamento deste suplemento possa ser realizado com maior certeza dos seus efeitos adversos e potenciais benefícios para a saúde.

\section{REFERÊNCIAS BIBLIOGRÁFICAS}

1. Chew KY, Brownlee IA. The impact of supplementation with dietary fibers on weight loss: A systematic review of randomised controlled trials. Bioact Carbohydrates Diet Fibre [Internet]. 2018;14(July):9-19. Available from: http://dx.doi.org/10.1016/j. bcdf.2017.07.010.

2. McRae MP. Effectiveness of Fiber Supplementation for Constipation, Weight Loss, and Supporting Gastrointestinal Function: A Narrative Review of Meta-Analyses. J Chiropr Med [Internet]. 2020;19(1):58-64. Available from: https://doi.org/10.1016/j. jcm.2019.10.008.

3. Devaraj RD, Reddy CK, Xu B. Health-promoting effects of konjac glucomannan and its practical applications: A critical review. Int J Biol Macromol [Internet]. 2019;126:27381. Available from: https://doi.org/10.1016/j.jjbiomac.2018.12.203.

4. Tester R, Al-Ghazzewi F. Glucomannans and nutrition. Food Hydrocoll [Internet]. 2017;68:246-54. Available from: http://dx.doi.org/10.1016/j.foodhyd.2016.05.017.

5. Panel E, Nda A. Scientific Opinion on the substantiation of health claims related to konjac mannan (glucomannan) and reduction of body weight (ID 854, 1556, 3725), reduction of post-prandial glycaemic responses (ID 1559), maintenance of normal blood glucose concentration. EFSA J. 2010;8(10).

6. Commission Regulation (EU) No 432/2012 of 16 May 2012, establishing a list of permitted health claims made on foods, other than those referring to the reduction of disease risk and to children's development and health, OJ L 136, 1-40.
7. Khan K, Jovanovski E, Ho HVT, Marques ACR, Zurbau A, Mejia SB, et al. The effect of viscous soluble fiber on blood pressure: A systematic review and meta-analysis of randomized controlled trials. Nutr Metab Cardiovasc Dis. 2018;28(1):3-13.

8. Zalewski BM, Chmielewska A, Szajewska $H$. The effect of glucomannan on body weight in overweight or obese children and adults: A systematic review of randomized controlled trials. Nutrition. 2015;31(3):437-442.e2.

9. Karri S, Sharma S, Hatware K, Patil K. Natural anti-obesity agents and their therapeutic role in management of obesity: A future trend perspective. Biomed Pharmacother [Internet]. 2019;110(November 2018):224-38. Available from: https:// doi.org/10.1016/j.biopha.2018.11.076.

10. Astell KJ, Mathai ML, SuXQ. Plant extracts with appetite suppressing properties for body weight control: A systematic review of double blind randomized controlled clinical trials. Complement Ther Med [Internet]. 2013;21(4):407-16. Available from: http://dx.doi.org/10.1016/j.ctim.2013.05.007.

11. Li B, Shah BR, Wang L, Liu S, Li Y, Wei X, et al. Health benefits of konjac glucomannan with special focus on diabetes. Bioact Carbohydrates Diet Fibre [Internet]. 2015;5(2):179-87. Available from: http://dx.doi.org/10.1016/j.bcdf.2015.03.007.

12. Zalewski BM, Szajewska H. No Effect of Glucomannan on Body Weight Reduction in Children and Adolescents with Overweight and Obesity: A Randomized Controlled Trial. J Pediatr. 2019;211:85-91.e1.

13. Zhu F. Modifications of konjac glucomannan for diverse applications. Food Chem. 2018;256(February):419-26.

14. Maia-Landim A, Ramírez JM, Lancho C, Poblador MS, Lancho JL. Long-term effects of Garcinia cambogia/Glucomannan on weight loss in people with obesity, PLIN4, FTO and Trp64Arg polymorphisms. BMC Complement Altern Med. 2018;18(1):1-9.

15. Martino F, Puddu PE, Pannarale G, Colantoni C, Martino E, Niglio T, et al. Low dose chromium-polynicotinate or policosanol is effective in hypercholesterolemic children only in combination with glucomannan. Atherosclerosis [Internet]. 2013;228(1):198-202. Available from: http://dx.doi.org/10.1016/j.atherosclerosis.2013.02.005.

16. Keithley JK, Swanson B, Mikolaitis SL, Demeo M, Zeller JM, Fogg L, et al. Safety and efficacy of glucomannan for weight loss in overweight and moderately obese adults. J Obes. 2013;2013.

17. Mohammadpour S, Amini MR, Shahinfar H, Tijani AJ, Shahavandi M, Ghorbaninejad P, et al. Effects of glucomannan supplementation on weight loss in overweight and obese adults: A systematic review and meta-analysis of randomized controlled trials. Obes Med [Internet]. 2020;19:100276. Available from: https://doi.org/10.1016/j. obmed.2020.100276.

18. Onakpoya I, Posadzki P, Ernst E. The Efficacy of Glucomannan Supplementation in Overweight and Obesity: A Systematic Review and Meta-Analysis of Randomized Clinical Trials. J Am Coll Nutr. 2014;33(1):70-8.

19. Jakopin Ž. Risks associated with fat burners: A toxicological perspective. Food Chem Toxicol [Internet]. 2019;123:205-24. Available from: https://doi.org/10.1016/j. fct.2018.10.051.

20. Kaats GR, Bagchi D, Preuss HG. Konjac Glucomannan Dietary Supplementation Causes Significant Fat Loss in Compliant Overweight Adults. J Am Coll Nutr. 2015;(October):1-7.

21. Ejtahed HS, Soroush AR, Siadat SD, Hoseini-Tavassol Z, Larijani B, HasaniRanjbar S. Targeting obesity management through gut microbiota modulation by herbal products: A systematic review. Complement Ther Med [Internet]. 2019;42:184-204. Available from: https://doi.org/10.1016/j.ctim.2018.11.019. 